\title{
Septic Arthritis of the Sternoclavicular Joint
}

Jason Womack, MD

Septic arthritis is a medical emergency that requires immediate action to prevent significant morbidity and mortality. The sternoclavicular joint may have a more insidious onset than septic arthritis at other sites. A high index of suspicion and judicious use of laboratory and radiologic evaluation can help solidify this diagnosis. The sternoclavicular joint is likely to become infected in the immunocompromised patient or the patient who uses intravenous drugs, but sternoclavicular joint arthritis in the former is uncommon. This case series describes the course of 2 immunocompetent patients who were treated conservatively for septic arthritis of the sternoclavicular joint. (J Am Board Fam Med 2012;25: 908-912.)

Keywords: Case Reports, Septic Arthritis, Sternoclavicular Joint

\section{Case 1}

A 50-year-old man presented to his primary care physician with a 1-week history of nausea, vomiting, and diarrhea. His medical history was significant for 1 episode of pseudo-gout. He had no chronic medical illnesses. He was noted to have a heart rate of 60 beats per minute and a blood pressure of $94 / 58 \mathrm{~mm} \mathrm{Hg}$. His heart rate increased to 72 beats per minute while seated. He had presumed gastroenteritis. He was given $2 \mathrm{~L}$ intravenous $0.9 \%$ normal saline in the outpatient setting, with an increase in blood pressure to $100 / 68 \mathrm{~mm}$ $\mathrm{Hg}$. He was seen 2 days later and was beginning to feel better but had a new complaint of tenderness at the left sternum. The patient began a 5-day course of prednisone. Blood was collected at this time. The next day, blood analysis revealed a critical laboratory value for creatine phosphokinase level of $9005 \mathrm{U} / \mathrm{L}$ and an erythrocyte sedimentation rate of $120 \mathrm{~mm} /$ hour. The patient was asked to go to the hospital to receive further evaluation. At the time

This article was externally peer reviewed.

Submitted 14 June 2011; revised 27 November 2011; accepted 5 December 2011.

From the Department of Family and Community Medicine, University of Medicine and Dentistry of New JerseyRobert Wood Johnson Medical School, New Brunswick, NJ.

Funding: none.

Conflict of interest: none declared.

Corresponding author: Jason P. Womack, MD, UMDNJRobert Wood Johnson Medical School, 1 Robert Wood Johnson Pl, MEB 2nd Floor, New Brunswick, NJ 08903 (E-mail: womackja@umdnj.edu). of admission, he continued to complain of left clavicular pain, and the course of prednisone failed to provide any pain relief. The patient denied any current fevers or chills. He was afebrile, and examination revealed a swollen and tender left sternoclavicular (SC) joint. The prostate was normal in size and texture and was not tender during palpation. Laboratory analysis showed a white blood cell count of 19.3 thousand/microliter, with $92 \%$ segmented neutrophils and no bands (Table 1). Urinalysis was significant for blood and leukocyte esterase. Blood and urine cultures were obtained. A radiograph of the clavicle was normal. The patient was started on intravenous fluids for the elevated creatine phosphokinase level. Ceftriaxone $(1 \mathrm{~g}$ intravenous daily) was started for the leukocytosis, pyuria, and history of fever. On hospital day 1, blood and urine cultures yielded Gram-negative bacilli that were later identified as Escherichia coli. It was believed that the E. coli bacteremia seeded the SC joint, leading to septic arthritis. A computed tomography (CT) scan of the sternum was performed to assist with diagnosis and showed no fluid in the SC joint and no sign of bony erosion or sclerosis at the manubrium or medial end of the clavicle. The antibiotic was changed to intravenous ciprofloxacin for better tissue penetration of the urinary tract because the prostate was a concerning source of infection and to provide adequate bone coverage for presumed septic arthritis of the SC joint.

The patient was discharged in good condition on hospital day 4 , and follow-up was arranged with 
Table 1. Blood Analysis for Patient in Case 1

\begin{tabular}{lcc}
\hline Test & Value & Normal \\
\hline WBC, $10^{3} / \mathrm{uL}$ & 19.3 & $4.0-10.0$ \\
Hemoglobin, g/dL & 11.4 & $14.1-17.7$ \\
Platelets, $10^{3} / \mathrm{uL}$ & 446 & $140-440$ \\
Sodium, mEq/L & 138 & $136-145$ \\
Potassium, mEq/L & 4.1 & $3.5-5.0$ \\
Bicarbonate, mEq/L & 26.8 & $24.0-32.0$ \\
Chlorine, mEq/L & 103 & $98-108$ \\
BUN, mg/dL & 22 & $6-23$ \\
Creatinine, mg/dL & 1.1 & $0.5-1.2$ \\
Glucose, mg/dL & 156 & $70-100$ \\
AST, IU/L & 305 & $12.0-45.0$ \\
ALT, IU/L & 140 & $3.0-40.0$ \\
Alkaline phosphatase, IU/L & 55 & $37-107$ \\
CPK, U/L & 10872 & $25-210$ \\
\hline
\end{tabular}

ALT, alanine aminotransferase; AST, aspartate aminotransferase; BUN, blood urea nitrogen, $\mathrm{CPK}$, creatine phosphokinase; WBC, white blood cells.

his primary care physician. He was discharged with an additional 4-week course of oral ciprofloxacin therapy. He returned to his primary care physician 2 weeks after discharge with continued complaints of left SC pain. Magnetic resonance imaging (MRI) showed bone marrow edema and signs of subacute septic arthritis and osteomyelitis (Figures 1 and 2). An orthopedic surgeon was consulted. Conservative treatment with anti-inflammatory medication was

Figure 1. Magnetic resonance image of the sternoclavicular joint for patient in case 1 showing edema in the manubrium and distal clavicle.

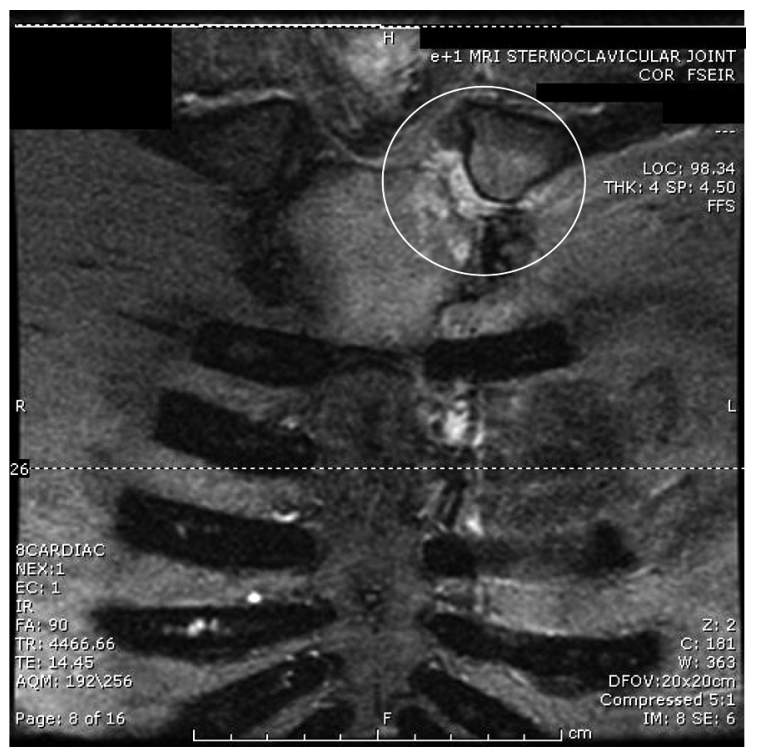

Figure 2. Magnetic resonance image of the sternoclavicular joint for patient in case 1 showing a sternoclavicular joint effusion and erosion of the manubrium and distal clavicle.

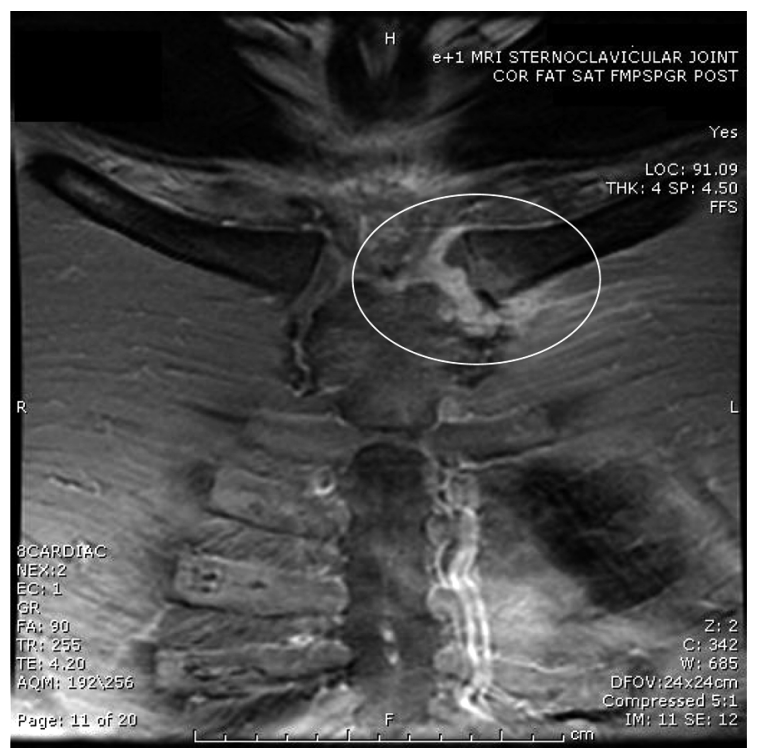

recommended because adequate antibiotic treatment had been given for both septic arthritis and osteomyelitis. The patient gradually began to notice improvement of the pain. Eight weeks after hospital discharge, the patient was pain free without the use of anti-inflammatory medications.

\section{Case 2}

A 69-year-old man with a history of prostate cancer treated with radioactive bead therapy had problems with recurrent bouts of urinary retention, which ultimately required a suprapubic catheter. It was removed 6 weeks before admission. He presented to the emergency department with 5 days of fever at $101.4^{\circ} \mathrm{F}$. He was seen by his physician, who prescribed ciprofloxacin secondary to symptoms of dysuria, polyuria, and urinary urgency. Symptoms continued despite 4 days of therapy, prompting his visit to the emergency department. In addition to urinary symptoms, the patient had severe pain in his right shoulder and anterior chest. Examination was significant for a temperature of $102.5^{\circ} \mathrm{F}$, a heart rate of 139 beats per minute, and a blood pressure of 126/66 mm Hg. The patient had suprapubic tenderness and bilateral costovertebral angle tenderness. His medical history was significant for type 2 diabetes mellitus, and he had been taking glipizide, sitagliptin, and enalapril. A recent glyco- 
sylated hemoglobin level was $6.8 \%$. Urinalysis was significant for nitrates, $3+$ leukocyte esterase, and $3+$ blood. Blood urea nitrogen and creatinine levels were $42 \mathrm{mg} / \mathrm{dL}$ and $2.1 \mathrm{mg} / \mathrm{dL}$, respectively. His white blood cell count was 24.3 thousand/ microliter. Physical examination was significant for swelling of the right SC joint, with tenderness during palpation. There was right chest pain with right shoulder motion. The patient was admitted and given ceftriaxone to treat sepsis from a urinary tract infection. On hospital day 1, the patient continued to have a fever. Blood cultures and urine cultures yielded $\mathrm{G}+$ cocci in clusters that eventually were identified as methicillin-resistant Staphylococcus aureus. Vancomycin was started and the ceftriaxone was discontinued. Because of the swelling of the SC joint, studies to evaluate that area were performed. $\mathrm{CT}$ and radiographs were negative for SC joint pathology. MRI showed a septic right SC joint and osteomyelitis of the manubrium. A triple-phase bone scan showed increased uptake at the right SC joint (Figure 3).

A peripherally inserted central catheter was placed, and the patient was discharged to complete an 8 -week course of intravenous antibiotics for septic arthritis of the SC joint and osteomyelitis of the manubrium and clavicle. The patient continued the antibiotics for 4 weeks, but they were discontinued because of elevated serum levels of vancomycin and concern about renal toxicity. The chest pain never com-

Figure 3. Delayed images of triple-phase bone scan in patient in case 2.

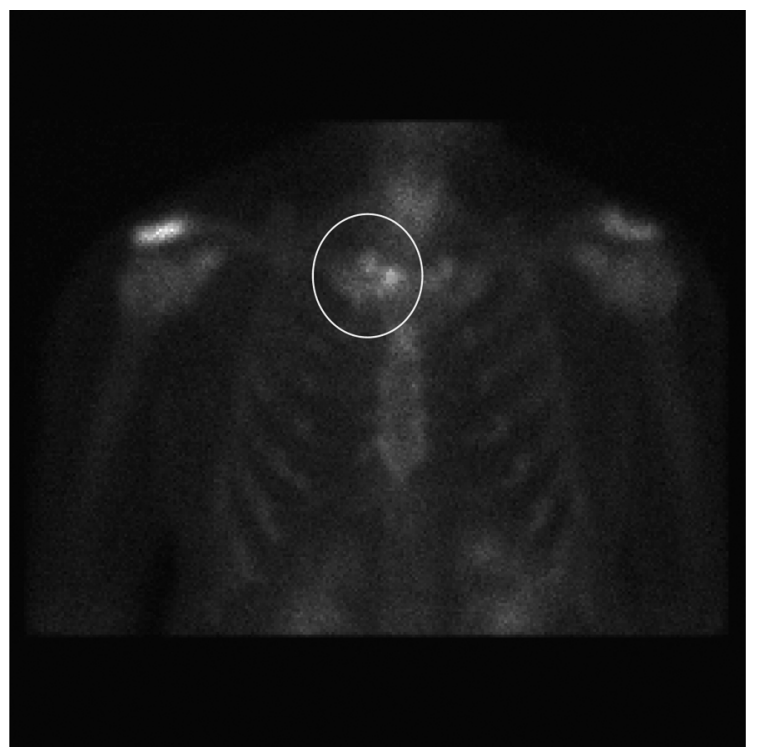

pletely abated, and the patient returned to the hospital 1 month later with continued swelling and erythema of the SC joint. Repeat MRI at that time showed continued osteomyelitis of the distal right clavicle and manubrium with septic arthritis of the SC joint. The patient was started on intravenous vancomycin for an additional 8 weeks of outpatient treatment. Drug levels and dosing were monitored closely to prevent the potentially harmful serum concentrations of vancomycin that caused premature discontinuation of the first round of antibiotic therapy.

\section{Discussion}

Septic arthritis is a medical emergency, with a mortality rate of $10 \% .{ }^{1}$ Even in the presence of treatment there is a $32 \%$ risk of substantial morbidity, including osteomyelitis and poor functional outcome. ${ }^{2}$ The large joints of the hip and the knee are most commonly affected in more than half of cases, but any joint can be involved. ${ }^{3}$ Most cases of septic arthritis occur in the setting of a joint with underlying structural pathology such as osteoarthritis, rheumatoid arthritis, or crystalline arthropathy. ${ }^{3}$ The SC joint is less commonly associated with septic arthritis but presents its own unique set of risk factors and complications.

Septic arthritis of the SC joint represents $1 \%$ of all bone and joint infections, but it is uncommon in healthy adults, representing less than $0.5 \%$ of bone and joint infections. ${ }^{4,5}$ It often is overlooked and has an insidious onset. ${ }^{5-7}$ Diabetes, rheumatoid arthritis, intravenous drug use, subclavian vein catheter placement, and other illnesses are risk factors for infection of the SC joint, but its presence in a healthy individual is rarely reported. It may occur from hematogenous spread from a distant source or from contiguous spread from a nearby infection. Any patient complaining of unilateral SC joint pain must be considered to have an infection until proven otherwise.

The SC joint is a diarthrodial joint and sits in a superficial position on the anterior chest wall. It is this location that predisposes the SC joint to an array of traumatic injuries and early recognition of pathology due to obvious swelling. Immediately posterior to the SC joint lie the great vessels, trachea, esophagus, vagus, and phrenic nerves. This proximity can cause substantial complications during trauma or surgery involving the SC joint and 
play a role in the propensity of the SC joint for infection when there is associated bacteremia. When pathology of the SC joint is suspected, imaging and laboratory studies should be performed to confirm the presence of disease.

Clinical presentation and laboratory analysis can provide clues about the underlying infection of the SC joint. Fever is present in $65 \%$ cases, and the patient is likely to complain of chest or shoulder pain. ${ }^{4}$ Approximately $56 \%$ of patients with an infection of the SC joint will be found to have a leukocytosis on complete blood count, and 62\% will have positive blood cultures. ${ }^{5}$ This information helps to support a presumptive diagnosis of septic arthritis of the SC joint and may prompt further diagnostic testing.

Radiologic studies of the SC joint help to further delineate underlying pathology. Plain radiographs may show sclerosis at the medical end of clavicle. This nonspecific sign may suggest on underlying osteoarthritis, SC septic arthritis, or systemic inflammatory process. ${ }^{8}$ Because of the limited information that plain radiographs provide, MRI, CT, and ultrasound may play a role in specifying the diagnosis. CT may be preferred for its higher availability and shorter time to perform the test. The earliest findings seen on CT or MRI are effusion, widening of the joint space, or mild cortical irregularity. Osteomyelitis of the distal clavicle, the manubrium, or both may be present in up to $55 \%$ of cases. ${ }^{5,8}$ Ultrasound has increasing utility in the diagnosis of musculoskeletal conditions when used by an experienced clinician. In the SC joint, findings such as effusion and irregularity of the bony margins may support a clinical notion of infection and assist in aspiration of the joint. ${ }^{9}$

Once radiologic studies support the diagnosis of septic arthritis of the SC joint, an attempt to aspirate fluid from the joint should be attempted to establish the microbial etiology. This may be difficult because of the paucity of fluid found in the small SC joint and the presence of an intra-articular disk. $^{6}$ In a review of 180 cases of SC joint pyoarthritis, only 65 aspirates were obtained. ${ }^{5}$ Peripheral blood cultures may isolate a particular microbe that can be assumed to be the offending agent in the SC joint. The most common isolate found in SC septic arthritis is S. aureus. ${ }^{5}$ Neisseria gonorrbea should be considered in people at risk for this sexually transmitted infection. Gram-negative bacteria such as Pseudomonas spp. and E. coli are less common but should be considered in patients with immunosuppression or concurrent peripheral infection.

Initial antibiotic treatment should be empiric parental therapy. It may be guided by gram stain of aspirated synovial fluid, but caution should be exercised because this identifies the causative organism in only $50 \%$ of cases. ${ }^{2}$ Therapy may be refined once cultures of synovial fluid or peripheral blood are available. An antistaphylococcal penicillin, such as oxacillin, is an adequate first choice in an otherwise healthy patient. Any history that would put the patient at risk for Gram-negative infection should prompt the use of broad-spectrum antibiotics until culture results are available. Antibiotic therapy should be tailored and continued for 4 weeks for septic arthritis. If there is concomitant osteomyelitis, which commonly occurs in the setting of SC septic arthritis, therapy should be continued for 6 weeks. ${ }^{5}$

The management of septic arthritis after initiation of parenteral antibiotics involves surgical drainage or repeated aspiration of the infected joint. ${ }^{10}$ This approach is difficult with the SC joint. There is often very little fluid to aspirate, and incision and drainage often has poor outcomes. ${ }^{11}$ When no complications exist, initial therapy for SC pyoarthrosis is longterm parenteral antibiotics. In the presence of immunosuppression, extensive osteomyelitis, or softtissue complication such as abscess or mediastinitis, surgical management with extensive debridement and resection of the medial clavicle is indicated. ${ }^{12}$ One may consider surgical options if the patient fails to respond to conservative measures within 48 hours. ${ }^{13}$ Surgery should be performed by an experienced thoracic surgeon. The proximity of the SC joint to many vital structures increases the risk of morbidity associated with surgery.

Local complications of arthritis in the SC joint can lead to more complicated disease. This infection may lead to the formation of a local abscess, mediastinitis, and superior vena cava syndrome. ${ }^{14}$ Abscesses may migrate and infect the surrounding soft tissues. ${ }^{15,16}$ Mediastinitis is the most serious complication but occurs in less than $15 \%$ of cases. Osteomyelitis of the sternum or the clavicle is one of the most common complications of SC joint arthritis, occurring in more than $50 \%$ of cases. $^{5,6}$

SC joint arthritis in healthy adults is an uncommon occurrence. Intravenous drug users have the highest risk of SC joint infection. ${ }^{5}$ Other risk fac- 
tors include diabetes mellitus and immunosuppressive diseases or use of immunosuppressant drugs. ${ }^{5}$ Placement of a subclavian central venous catheter can lead to an infection in the SC joint. This may be from bacteremia of an infected indwelling catheter or from direct inoculations from repeated attempts at percutaneous cannulation. ${ }^{7}$

\section{Conclusions}

Septic arthritis of the SC joint in immunocompetent patients who are not using intravenous drugs is a rarely reported event. It most commonly occurs in the setting of intravenous drug use or in a person with an underlying immunocompromised state. ${ }^{5} E$. coli and S. aureus bacteremia from a urinary source seemed to be the causes of septic arthritis of the SC joints in our case series. When a patient complains of unilateral SC pain, further evaluation should commence to rule out an infectious process. The small characteristics of the SC joint make diagnosis more difficult than septic arthritis of a larger joint. Radiologic studies and peripheral laboratory analysis can support the analysis, but it may be difficult to obtain a definitive bacterial diagnosis. Only a small percentage of cases were able to provide synovial fluid for analysis. Conservative treatment with long-term parenteral antibiotics is the treatment of choice. Some patients may require surgical intervention if they fail conservative measures.

\section{References}

1. Gupta MN, Sturrock RD, Field M. A prospective 2-year study of 75 patients with adult-onset septic arthritis. Rheumatology 2001;40:24-30.

2. Weston VC, Jones AC, Bradbury N, Fawthrop F, Doherty M. Clinical features and outcome of septic arthritis in a single UK Health District 1982-1991. Ann Rheum Dis 1999;58:214-9.
3. Mathews CJ, Coakley G. Septic arthritis: current diagnostic and therapeutic algorithm. Curr Opin Rheum 2008;20:457-62.

4. Yood RA, Goldenberg DL. Sternoclavicular joint arthritis. Arthritis Rheum 1980;23:232-9.

5. Ross JJ, Shamsuddin H. Sternoclavicular septic arthritis: review of 180 cases. Medicine 2004;83:139-48.

6. Bar-Natan M, Salai M, Sidi Y, Gur H. Sternoclavicular infectious arthritis in previously healthy adults Semin Arthritis Rheum 2002;32:189-95.

7. Pradhan C, Watson NF, Jagasia N, Chari R, Patterson JE. Bilateral sternoclavicular joint septic arthritis secondary to indwelling central venous catheter: a case report. J Med Case Rep 2008;2:131.

8. Harden SP, Argent JD, Blaquiere RM. Painful sclerosis of the medial end of the clavicle. Clin Radiol 2004;59:992-9.

9. Ernberg LA, Potter HG. Radiographic evaluation of the acromioclavicular and sternoclavicular joints. Clin Sports Med 2003;22:255-75.

10. Ross JJ, Saltzman CL, Carling P, Shapiro DS. Pneumococcal septic arthritis: review of 190 cases. Clin Infect Dis 2003;36:319-27.

11. Song HK, Guy TS, Kaiser LR, Shrager JB. Current presentation and optimal surgical management of sternoclavicular joint infections. Ann Thorac Surg 2002;73:427-31.

12. Burkhart HM, Deschamps C, Allen MS, Nichols FC 3rd, Miller DL, Pairolero PC. Surgical management of sternoclavicular joint infections. J Thorac Cardiovasc Surg 2003;125:945-9.

13. Robinson CM, Jenkins PJ, Markham PE, Beggs I. Disorders of the sternoclavicular joint. J Bone Joint Surg Br 2008;90:685-96.

14. Koroscil TM, Valen PA. Sternoclavicular septic arthritis due to Haemophilus influenzae. South Med J 1990;83:1469-71.

15. Cone LA, Lopez C, O'Connell SJ, Nazemi R, Sneider RE, Denker H. Staphylococcal septic synovitis of the sternoclavicular joint with retrosternal extension. J Clin Rheumatol 2006;12:187-9.

16. Dhulkotia A, Asumu T, Solomon P. Breast abscess: a unique presentation as primary septic arthritis of the sternoclavicular joint. Breast J 2005;11:525-6. 